\title{
Forecasting model of COVID-19 pandemic in Malaysia: An application of time series approach using neural network
}

\author{
Titi Purwandaria ${ }^{a}$, Solichatus Zahroha ${ }^{a}$, Yuyun Hidayat ${ }^{a}$, Sukono ${ }^{b}$, Mustafa Mamat ${ }^{\mathfrak{c}}$ and Jumadil \\ Saputra $^{\mathrm{d}^{*}}$
}

${ }^{a}$ Department of Statistics, Faculty of Mathematics and Natural Sciences, Universitas Padjadjaran, Indonesia

${ }^{b}$ Department of Mathematics, Faculty of Mathematics and Natural Sciences, Universitas Padjadjaran, Indonesia

${ }^{c}$ Faculty of Informatics and Computing, Universiti Sultan Zainal Abidin, Terengganu, Malaysia, 22000 Besut, Terengganu, Malaysia ${ }^{d}$ Faculty of Business, Economics and Social Development, Universiti Malaysia Terengganu, 21030 Kuala Nerus, Terengganu, Malaysia

\section{H R O N I C L E}

Article history:

Received July 5, 2021

Received in revised format:

September 1, 2021

Accepted October 82021

Available online

October 9, 2021

Keywords:

Forecasting model

COVID-19 pandemic

Movement control order

Neural Network

Malaysia

\begin{abstract}
A B S T R A C T
COVID-19 has spread to more than a hundred countries worldwide since the first case reported in late 2019 in Wuhan, China. As one of the countries affected by the spread of COVID-19 cases, the local government of Malaysia has issued several policies to reduce the spread of this outbreak. One of the measures taken by the Malaysian government, namely the Movement Control Order, has been carried out since March 18, 2020. In order to provide precise information to the government so that it can take the appropriate measures, many researchers have attempted to predict and create the model for these cases to identify the number of cases each day and the peak of this pandemic. Therefore, hospitals and health workers can anticipate a surge in COVID19 patients. In this research, confirmed, recovered, and death cases prediction was performed using the neural network as one of the machine learning methods with high accuracy. The neural network model used is the Multi-Layer Perceptron, Neural Network Auto-Regressive, and Extreme Learning Machine. The three models calculated the average percentage error (APE) values for 7 days and obtained APE values for most cases less than $10 \%$; only 1 case in the last day of one method had an APE value of approximately $11 \%$. Furthermore, based on the best model, then the forecast is made for the next 7 days. In conclusion, this study identified that the MLP model is the best model for 7-step ahead forecasting for confirmed, recovered, and death cases in Malaysia. However, according to the result of testing data, the ELM performs better than the MLP model.
\end{abstract}

(C) 2022 by the authors; licensee Growing Science, Canada.

\section{Introduction}

The COVID-19 disease started in Wuhan, China, as of the end of December 2020. On June 15, 2020, this outbreak spread to 215 countries and territories, also affecting 8,128,490 people, with 439,421 deaths (Worldometer, 2020). Due to the severity of the virus, on January 30, 2020, the World Health Organization (WHO) declared that the COVID-19 epidemic had become a global health emergency of international concern. In Malaysia, the first case of COVID-19 was confirmed on January 25, 2020, involving three Chinese citizens who entered Johor Bharu, Malaysia, via Singapore on January 23, 2020 (Foo et al., 2020 ). Out of 32 million total population, Malaysia has reported 8,494 confirmed cases of COVID. -19 cases with 121 deaths in mid-June 2020. COVID-19 spread through direct or indirect contact, droplet spray for short-range transmission, and aerosol for longdistance transmission (Moriyama et al., 2020). In order to support the belief that the virus spreads between people in close contact with each other through respiratory droplets that arise when an infected person coughs, sneezes, or speaks, possibly from people through contact with contaminated surfaces or objects (CDC, 2019). COVID-19, thus, can also be spread by people

* Corresponding author.

E-mail address: jumadil.saputra@umt.edu.my (J. Saputra)

(C) 2022 by the authors; licensee Growing Science, Canada. doi: $10.5267 /$ j.ds1.2021.10.001 
who show no symptoms (Rettner, 2020). However, person-to-person transmission is an essential factor in the spread of this outbreak through any activity related to physical interactions (Dalton et al., 2020). As the number of deaths from global transmission through the community has increased, governments in several countries have put a barrier to control this pandemic, namely lockdown. The UK government estimates that mortality rates can increase rapidly and prevent social distance from being maintained (Mahase, 2020). Physical and social distance measurements aim to reduce the spread of disease by disrupting the chain of COVID-19 transmission and preventing the emergence of new ones. The initiative guarantees a physical distance between people (at least one meter) and minimizes face-to-face interaction while promoting and maintaining virtual social relationships within families and communities (WHO, 2020). However, government measures might overcome the other problems, such as mental health conditions, especially for the children (Pontoh et al., 2021a), economic stability in a country (Pontoh et al., 2021b).

To fully contain the virus, the Malaysian Prime Minister announced the four phases of the Movement Control Order (MCO) because they guessed that the number of COVID-19 cases would continue to increase (PMO, 2020) from March 18 to prevent widespread use (Arumugam, 2020). Figure 1 shows that the number of confirmed cases significantly increased day by day since early March. The ban during the MCO will continue to put pressure on the Malaysian tourism industry, and therefore effective measures to support industry players are crucial (Foo et al., 2020). The Malaysian government has also announced that it will avoid unnecessary public gatherings, including sporting, social, cultural, and religious events, and maintain social distance from others (Haque et al., 2020). The main focus of social distance is the physical distance to others, where public places such as supermarkets, bazaars, and shopping centers are to be avoided (FMT, 2020). Social alignment is a public health technique that people can use to slow the spread and spread of infectious diseases such as coronaviruses. These local measures had been done in Indonesia and worked effectively to control the pandemic's spread (Pontoh et al., 2020a).

Although the number of deaths reported in the country on the hyperbolic curve is not increasing (Fig. 1), there is an urgent need to be well prepared to deal with the deaths from this epidemic. It is important not only for health professionals but especially for first aiders. The positive COVID-19 cases active in Malaysia have continued to increase since March 2020. The National Institute of Forensic Medicine (IPFN) in Malaysia has to look to the worst-case scenario of a sudden increase in the number of deaths due to COVID-19 to prepare, which can exceed the capacity of forensic services (Khoo et al., 2020) because the number of confirmed cases is dramatically rising. Due to the intervention announced by the government, the number of death cases keeps steadily increasing to 100 cases (see Fig. 1). As of May 13, 2020, the number of deaths in Malaysia had only increased by 9 . Besides that, the cure rate of COVID-19 patients in Malaysia is quite high, i.e., 86\% as of June 13, 2020.

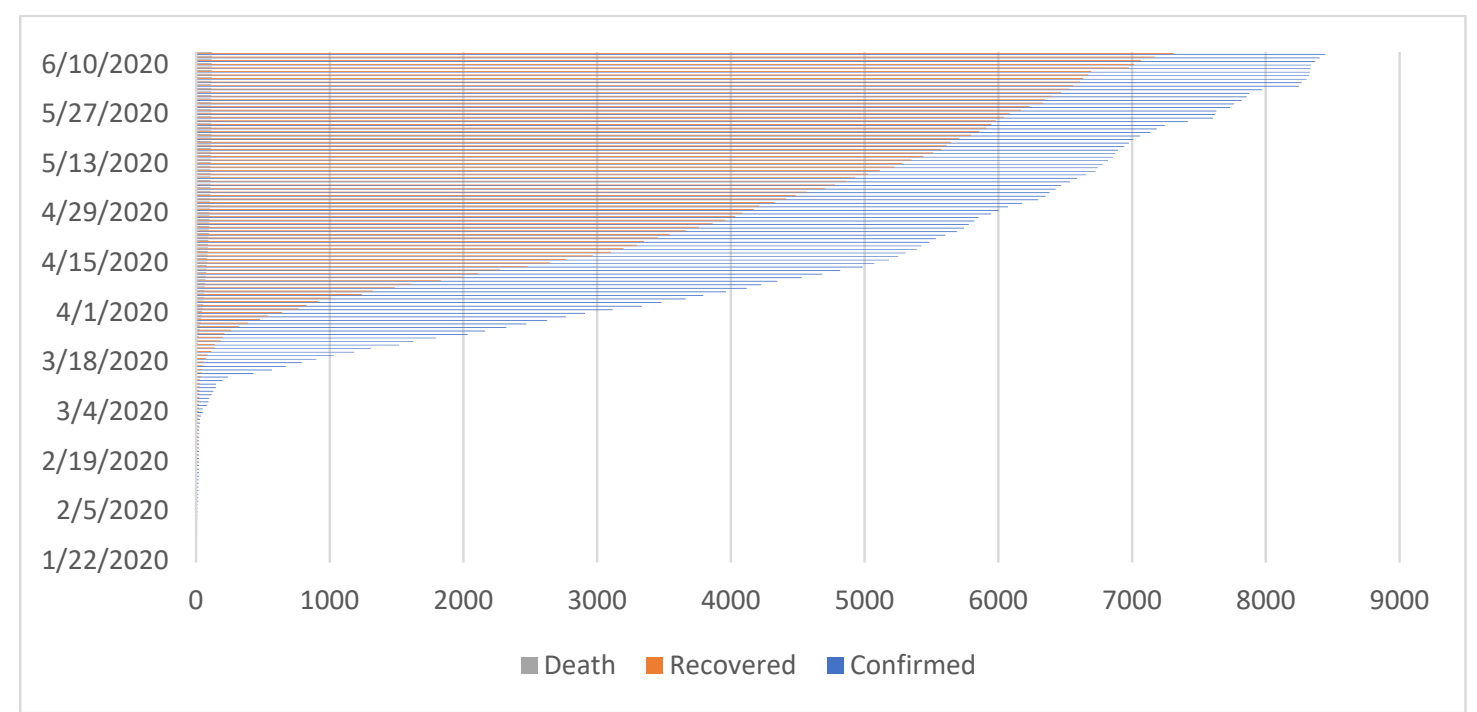

Fig. 1. The confirmed, recovered, and death cases in Malaysia

Note: January 22, 2020, to June 13, 2020

Many researchers worldwide tried to perform modeling also forecasting the number of this outbreak's cases and the peak of this disease. However, forecasting is more difficult due to the lack of past epidemiological information (Huang et al., 2020). In addition, interventions by the current government are varying the results of forecasting uncontrollably. This research aims to find the most appropriate model to predict the number of confirmed, recovered, and death cases in Malaysia. All models used in this study are simple models that do not consider other factors that can affect the development of these cases. In this study, we created a representation model for the number of COVID-19 cases using different neural network models, specifically Neural Network Auto-Regressive, Multi-Layer Perceptron, and Extreme Learning Machine. These models were used previously in South Korea and obtained good accuracy for seven-step ahead forecasting (Pontoh et al., 2020b). 


\section{Materials and Methods}

The historical data of the number of confirmed, recovered, and death cases of the COVID-19 pandemic in Malaysia would separate into training and testing. The training data are starting January 22, 2020, to June 6, 2020, and testing data starting June 7, 2020, to June 13, 2020. From the best model, then, forecasting results are generated for the next seven-day. The datasheet was available in Novel Coronavirus (COVID-19) Cases Data provided by Humanitarian Data Exchange.

\subsection{Artificial Neural Network (ANN)}

ANN, first introduced by McCulloch and Pits in 1943 (McCulloh and Pits, 1943), is an associate science system that is believed to be highly accurate (Fausset, 1994). ANN has three types of layers: the input layer, output layer, and hidden layer. ANN is divided into two types, Feed Forward Neural Network (FFNN) and Recurrent Neural Network (RNN). FFNN is a network where connections between neurons in a layer do not form cycles as the RNN performed, which means that the input only propagates forward from the input level to the output level (Toharudin et al., 2021).

\subsection{Multi-Layer Perceptron (MLP)}

The machinability in perceptron for single-layer feed-forward networks is targeted by (Rosenblatt 1962). FFNN is the foremost well-known neural network model for applications of time series prediction. It considers a single hidden layer with many neurons. A total of rehashes systems are fitted, each with random beginning weights that arrive at the midpoint of once processing forecasts.

Two types of FFNN are Single-Layer Perceptron (SLP) with no hidden layer and Multi-Layer Perceptron (MLP) with one or more hidden layers. From a statistical point of view, MLP's universal approximation ability arises from the nonlinearities used in the neurons (Du and Swamy, 2013). Every single connection between the input to neuron and neuron to the neuron is adjusted by weight. Also, every single neuron has an additional input, namely bias, that a persistent value of one is assumed (Plummer, 2000). Once the network is running, each neuron in a hidden layer carries out the computation on its inputs and conveys the result $\left(\mathrm{O}_{\mathrm{c}}\right)$ to the consecutive layer of the neurons.

$$
O_{c}=h_{\text {Hidden }}\left(\sum_{p-1}^{P} i_{c, p} w_{c, p}+b_{c}\right) \text { wherever } h_{\text {Hidden }}(x)=\frac{1}{1+e^{-x}} .
$$

Eq. (1) is an activation function of a neuron in a hidden layer. Where $O_{c}$ is that the output of the existing hidden layer neuron $c, P$ is the number of neurons among the earlier hidden layer or the network input, $i_{c, p}$ is input to neuron $c$ of the earlier hidden layer neuron $p, w_{c, p}$ is the weight that modifies the link from neuron $p$ to neuron $c$, and $b_{c}$ is the bias. In Eq. (1), $h_{H i d d e n}(x)$ is the sigmoid activation function of the neuron. Hence, the data should be scaled to avoid the network being more durable during the training process. In the same way, to fittingly scaled data before training, the weights and biases are initialized. Each neuron in the output layer carries out the subsequent computation equation on its inputs and transmits the outcome $\left(O_{c}\right)$ to a network output.

$$
O_{c}=h_{\text {Output }}\left(\sum_{p-1}^{P} i_{c, p} w_{c, p}+b_{c}\right) \text { wherever } h_{\text {Output }}(x)=x .
$$

Eq. (2) is the activation function of associate neurons in the output layer. Where $O_{c}$ is the output of the existing output layer neuron $c, P$ is the number of neurons among the earlier hidden layer, $i_{c, p}$ is the input to neuron $c$ from the earlier hidden layer neuron $p, w_{c, p}$ is the weight that modifies the link from neuron $p$ to neuron $c$, and $b_{c}$ is the bias. Activation function will be used for $h_{\text {Output }}(x)$ is a linear activation function.

\subsection{Neural Network Auto Regression Model (NNAR)}

A feed-forward neural network is fitted with slacked estimations of $y$ as inputs and a single hidden layer with extent neurons. The inputs are for lags 1 to $p$, and lags $m$ to MP where $m=f r e q(y)$. On the off chance that $x_{\text {reg }}$ is given, its columns are additionally utilized as inputs. However, if there are missing values in $y$ or $x_{\text {reg }}$ the relating rows are discarded from the fit. A sum of rehashes networks is fitted, each with irregular beginning weights. Once computing forecasts, at that point, these are found the middle value. The network processes multi-step predictions, although one-step predictions are prepared. The fitted model for data with a non-seasonal pattern is $\operatorname{NNAR}(p, k)$, where $k$ is the number of hidden neurons. It is practically equivalent to an $\operatorname{AR}(p)$ with non-linear functions. However, while linear AR can model cyclicity, the modeled cycles are always symmetric. In contrast, the cyclicity in the NNAR model has been modeled well and captured the asymmetry of the cycles. It is the one difference between AR and NNAR (Hyndmann \& Athanasopoulos, 2018).

\subsection{Extreme Learning Machine (ELM)}

ELM was proposed by Huang et al. (2004). ELM is a rapid learning algorithm for the single hidden layer feed-forward neural networks, overcomes the debility of the previous neural network in the process of learning speed because ELM could be reducing the training time and improving the generalization performance (Wang et al., 2014; Huang et al., 2006). The weights 
and biases of the hidden neurons are set randomly. Moreover, Moore-Penrose pseudoinverse (Liang and Huang, 2006) is used to determine the output weight under the criterion of the least-squares method (Xiao et al., 2017). For $N$ random definite samples $\left(x_{i}, t_{i}\right)$, wherever $x_{i}=\left[x_{i 1}, x_{i 2}, \ldots, x_{i n}\right]^{T} \in R^{n}$ and $t_{i}=\left[t_{i 1}, t_{i 2}, \ldots, t_{i n}\right]^{T} \in R^{m}$, more, $\left(x_{i}, t_{i}\right) \in R^{n} x R^{m}$ and $i=$ $1,2, \ldots, N$. Standard ELM with $\widetilde{N}$ hidden neurons and activation function $f(x)$ are computationally model as

$$
\sum_{i=1}^{\widetilde{N}} \beta_{i} f_{i}\left(x_{j}\right)=\sum_{i=1}^{\widetilde{N}} \beta_{i} f\left(a_{i} \cdot x_{j}+b_{i}\right)=t_{j} \text { with } j=1,2, \ldots, N,
$$

where $a_{i}=\left[a_{i 1}, a_{i 2}, \ldots, a_{i n}\right]^{T}$ is the weight vector that linking the $i^{\text {th }}$ hidden and input neurons, $b i$ is the threshold of the $i^{\text {th }}$ hidden neurons, $\beta_{i}=\left[\beta_{i 1}, \beta_{i 2}, \ldots, \beta_{i m}\right]^{T}$ is the weight vector linking the $i^{\text {th }}$ hidden and output neurons. The activation function that used commonly is sigmoid, sine, and RBF. Equation 3 also can be written as $H \beta=T$ where:

$$
\boldsymbol{H}\left(a_{1}, \ldots, a_{\widetilde{N}}, b_{1}, \ldots, b_{\widetilde{N}}, x_{1}, \ldots, x_{N}\right)=\left[\begin{array}{ccc}
f\left(a_{1} \cdot x_{1}+b_{1}\right) & \cdots & f\left(a_{\widetilde{N}} \cdot x_{1}+b_{\widetilde{N}}\right) \\
\vdots & \ddots & \vdots \\
f\left(a_{1} \cdot x_{N}+b_{1}\right) & \cdots & f\left(a_{\widetilde{N}} \cdot x_{N}+b_{\widetilde{N}}\right)
\end{array}\right]_{N \times \widetilde{N}}, \boldsymbol{\beta}=\left[\begin{array}{c}
\beta_{1}^{T} \\
\vdots \\
\beta_{\widetilde{N}}^{T}
\end{array}\right]_{\widetilde{N} \times m}, \boldsymbol{T}=\left[\begin{array}{c}
t_{1}^{T} \\
\vdots \\
t_{\widetilde{N}}^{T}
\end{array}\right]_{N \times m}
$$

$H$ is the so-called hidden layer output matrix of the network, the $i^{\text {th }}$ column of $H$ is the $i^{\text {th }}$ hidden neuron output concerning inputs. ELM allocates to the input link weights $a$ and hidden layer neuron biases $b$ arbitrarily when the training starts. While the training process is unvaried, it could approach any continuous function. As a means to gain better generalization performance, take $\widetilde{N}^{\prime \prime} N$. The input samples might obtain the hidden layer output matrix once the input weights and hidden layer biases are stubborn according to the arbitrary allocation. Accordingly, the training process of the ELM is broken down into solving linear equations $H \beta=T$ least-squares solution. The smallest norm least-squares solution of $H \beta=T$ is

$$
\widehat{\boldsymbol{\beta}}=\boldsymbol{H}^{\dagger} \boldsymbol{T} \text {. }
$$

$H^{\dagger}$ means Moore-Penrose pseudoinverse of the $H$. Generally, the optimal solution of $\hat{\beta}$ covers some characteristics. The optimal generalization ability of the minimum model of the output link weights and network. The optimal local solution might prevent to produce due to the exclusive of $\hat{\beta}$.

\subsection{Model Evaluation}

After obtaining a model for prediction from the training process, the model is evaluated with testing data to get the predicted accuracy value of the model every day. The testing data will be evaluated using Absolute Percentage Error every single day. Root Mean Square Error (RMSE) is widely used to assess error for the next seven-day when comparing methods with the same unit. Also, Mean Absolute Error (MAE) is used for the benchmark.

$$
\begin{aligned}
& A P E=\frac{\left|X_{t}-\hat{X}_{t}\right|}{X_{t}} \times 100 \% \\
& R M S E=\sqrt{\frac{1}{n} \sum_{t=1}^{n}\left(X_{t}-\hat{X}_{t}\right)^{2}} \\
& M A E=\frac{\sum_{t=1}^{n}\left|X_{t}-\hat{X}_{t}\right|}{n}
\end{aligned}
$$

where $n$ is the number of observations, $X_{t}$ is the observed value, and $\widehat{X_{t}}$ is the predicted value. A model can be said to be good if the value of APE is under $10 \%$, and the selected model for prediction is the model which has the smallest RMSE and MAE values.

\section{Results And Discussion}

The training data are starting January 22, 2020, to June 6, 2020, and testing data starting June 7, 2020, to June 13, 2020. First,

\begin{tabular}{|c|c|c|c|c|c|c|c|c|c|c|c|c|}
\hline \multirow{2}{*}{ Date } & \multicolumn{3}{|c|}{ NNAR $(1,1)$} & \multicolumn{3}{|c|}{ ELM } & \multicolumn{3}{|c|}{ MLP (5) } & \multicolumn{3}{|c|}{$\operatorname{MLP}(10,5)$} \\
\hline & $\mathrm{C}$ & $\mathrm{R}$ & $\mathrm{D}$ & $\mathrm{C}$ & $\mathrm{R}$ & $\mathrm{D}$ & $\mathrm{C}$ & $\mathrm{R}$ & $\mathrm{D}$ & $\mathrm{C}$ & $\mathrm{R}$ & $\mathrm{D}$ \\
\hline 07 June 2020 & 0.90 & 0.96 & 0.85 & 1.05 & 0.16 & 0.85 & 0.83 & 0.03 & 0.85 & 0.49 & 0.12 & 1.71 \\
\hline 08 June 2020 & 1.57 & 1.57 & 0.85 & 1.84 & 0.45 & 1.71 & 1.80 & 0.24 & 1.71 & 1.27 & 0.45 & 3.42 \\
\hline 09 June 2020 & 2.17 & 5.79 & 1.71 & 2.53 & 2.88 & 1.71 & 2.86 & 3.23 & 2.56 & 2.20 & 2.88 & 4.27 \\
\hline 10 June 2020 & 2.65 & 6.56 & 2.54 & 3.45 & 2.81 & 1.69 & 4.16 & 3.17 & 3.39 & 3.17 & 2.69 & 5.08 \\
\hline 11 June 2020 & 3.41 & 7.43 & 2.54 & 3.98 & 2.82 & 2.54 & 5.23 & 3.23 & 4.24 & 3.85 & 2.63 & 5.93 \\
\hline 12 June 2020 & 4.14 & 8.93 & 4.20 & 4.43 & 3.54 & 2.52 & 6.33 & 3.98 & 4.20 & 5.61 & 3.22 & 6.72 \\
\hline 13 June 2020 & 4.94 & 10.86 & 5.00 & 4.77 & 4.75 & 2.50 & 7.42 & 5.21 & 4.17 & 6.23 & 4.34 & 6.67 \\
\hline
\end{tabular}
the three methods explained before are used to train the data. Then, with the testing data, those models are generated and evaluate the values of APE every single day from June 7, 2020, to June 13, 2020. The APE values for each model are presented in Table 1.

Table 1

The percentage value of APE tested by using NNAR, ELM, and MLP models 
From the three models in Table 1, forecasting is performed. Then, the evaluation of the model is carried out for the forecasting for 7-step ahead. The value of metric evaluation for each case that contains RMSE and MAE is shown in Table 2.

Table 2

The value of metric evaluation using the NNAR, ELM, and MLP models

\begin{tabular}{ccccccc} 
& \multicolumn{2}{c}{ Confirmed } & \multicolumn{2}{c}{ Recovered } & Death & MAE \\
& RMSE & MAE & RMSE & 34.97 & 1.17 \\
NNAR & 60.91 & 48.81 & 50.64 & 27.51 & 1.14 \\
ELM & 40.50 & 28.80 & 38.46 & 13.44 & 0.74 \\
MLP (5) & $\mathbf{3 4 . 5 2}$ & $\mathbf{2 1 . 5 1}$ & 25.60 & $\mathbf{4 . 6 0}$ & 0.80 \\
MLP (10,5) & $\mathbf{3 4 . 5 2}$ & $\mathbf{2 1 . 5 1}$ & $\mathbf{7 . 9 8}$ & $\mathbf{0 . 6 6}$ & $\mathbf{0 . 3 2}$ \\
\hline
\end{tabular}

Table 2 shows the forecasting will be conducted using MLP $(10,5)$ for all the cases because this model has the lowest value of RMSE and MAE, even though MLP (5) and MLP (10,5) have the same values for the confirmed cases. However, as mentioned in Table 1, the minimum APE is produced by the ELM model. Therefore, we will forecast with both models, ELM and MLP (10,5). The interval forecasting results for the next 7-day, in other words, from June 14, 2020, to June 20, 2020, with a 5\% error from the point forecast, are mentioned in Table 3, and the plot of forecasting results can be seen in Fig. 2.

Table 3

The forecasting results of ELM and MLP models

\begin{tabular}{|c|c|c|c|c|c|c|}
\hline & \multicolumn{3}{|c|}{ ELM } & \multicolumn{3}{|c|}{$\operatorname{MLP}(10,5)$} \\
\hline & Confirmed & Recovered & Death & Confirmed & Recovered & Death \\
\hline 14 June 2020 & $8069-8919$ & $7008-7746$ & $115-127$ & $8070-8920$ & $7004-7742$ & $115-127$ \\
\hline 15 June 2020 & $8121-8975$ & $7076-7820$ & $116-128$ & $8123-8979$ & $7092-7838$ & $116-128$ \\
\hline 16 June 2020 & $8175-9035$ & $7148-7900$ & $117-129$ & $8177-9037$ & 7194-7952 & $117-129$ \\
\hline 17 June 2020 & 8233-9099 & $7225-7985$ & $117-129$ & $8237-9104$ & $7299-8067$ & $118-130$ \\
\hline 18 June 2020 & $8294-9167$ & $7286-8052$ & $118-130$ & $8297-9171$ & $7353-8127$ & 119-131 \\
\hline 19 June 2020 & $8357-9237$ & $7348-8122$ & $119-131$ & $8358-9238$ & $7498-8288$ & $120-132$ \\
\hline 20 June 2020 & 8423-9309 & $7409-8189$ & $120-132$ & $8418-9304$ & $7617-8419$ & $122-134$ \\
\hline
\end{tabular}

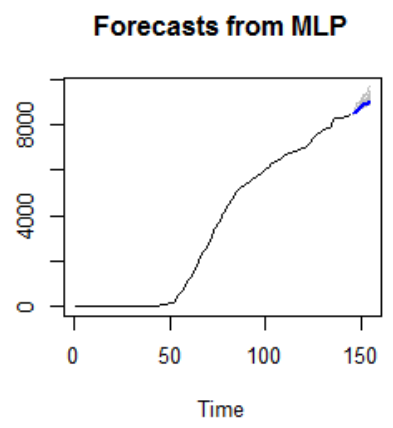

Forecasts from ELM

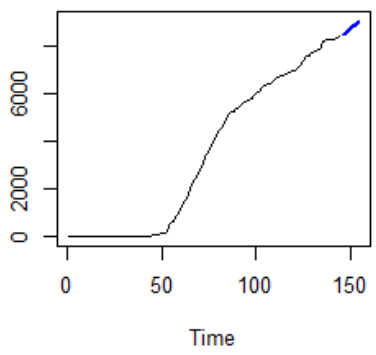

Forecasts from MLP

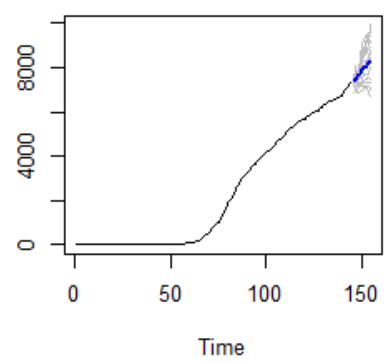

Forecasts from ELM

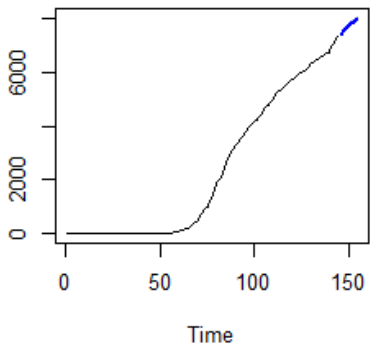

Forecasts from MLP

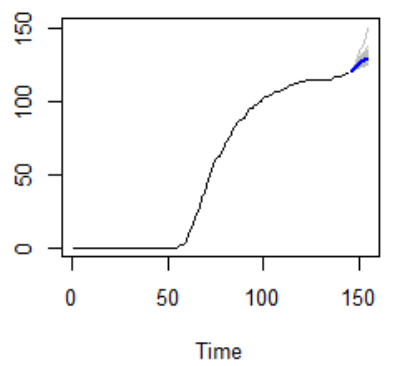

Forecasts from ELM

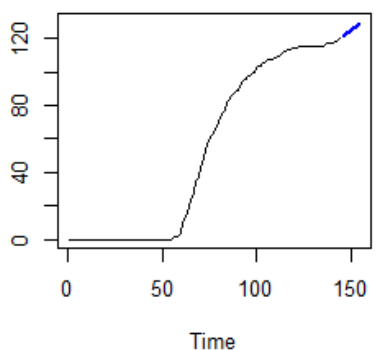

Fig. 2. Results of forecasting using ELM and MLP models

Note: Left to right: confirmed, recovered, deaths with y-axis is the number of cases, and the x-axis is the time period. 
The percentage value of APE was tested using NNAR, ELM, and MLP models that showed good accuracy. However, the APE values for all models are below $10 \%$. Only the NNAR model for recovered cases has a value above $10 \%$ on the seventh day. Additionally, all models will also be looking for the value of metric evaluation from the combined training and testing data for further prediction in seven days. The best model, then, forecasting results will be generated for the next seven-day. Before that, the characteristics of each model will be explained.

The NNAR method obtained a 1-1-1 network with 4 weights for confirmed, recovered, and death cases. First, it means that the order for both AR and NN is 1 with a difference. Secondly, the ELM method fitted 100 hidden neurons for all the cases with output weight using the lasso and combined the median operator. Lastly, the MLP has two models. On the one hand, MLP has a hidden layer fitted with 5 hidden neurons. Next, MLP with two hidden layers uses 10 hidden neurons in the first hidden layer and 5 hidden neurons in the second hidden layer. The number of hidden neurons is determined earlier. The ELM and MLP reached the optimum model after 20 repetitions with different univariate lags in each case. For the confirmed cases, the univariate lags are $(1,3)$. Univariate lags for the recovered cases are $(1,2,3,4)$ and the death cases, using univariate lags of $(2,3,4)$.

The lag values in the time series can be used as input for an artificial neural network.NNAR uses a single hidden layer for forecasting univariate time series data. Each hidden layer node performs a single sigmoid transformation of its input. The NNAR function produced a good model by averaging the results of 20 networks with linear output units, but it was not the best. A trial-and-error mechanism will produce a better model than the function automatically selected model (Toharudin et al., 2021). Based on Table 2, the forecasting will be conducted using MLP $(10,5)$ for all the cases because this model has the lowest value of RMSE and MAE, even though MLP (5) and MLP $(10,5)$ have the same values for the confirmed cases. However, as mentioned in Table 1, the minimum APE is produced by the ELM model. Therefore, to facilitate it, we will forecast with both models, ELM and MLP $(10,5)$. The interval forecasting results for the next 7-day, in other words, from June 14, 2020, to June 20, 2020, with a 5\% error from the point forecast.

\section{Conclusion}

In conclusion, this study identified that the MLP model is the best model for 7-step ahead forecasting for confirmed, recovered, and death cases in Malaysia. However, according to the result of testing data, the ELM performs better than the MLP model. It is possible that the evaluation of the model was carried out with different metrics. Nevertheless, the interval predictions are not significantly different between the two models. Also, the forecast results for confirmed cases in Malaysia did not increase substantially, or the curves tended to be sloping. It can, therefore, be concluded that the Malaysian government's interventions to manage COVID-19 have been fairly successful. It is certainly also influenced by the community's behavior, which carries out the government interventions well. Modeling the cases in Malaysia used in this study is a simple model without considering other factors that affect the number of these cases. The other factors are assumed to follow the existing pattern. In addition, the policy addition that was implemented by the local government to reduce the number of infected cases with COVID-19 also affected the outcome of the forecasts. Since forecasting the cases becomes hard, further research is needed to find out which factors influence the forecast value of the number of cases so that the forecast results might use another approach obtained more accurately.

\section{References}

Arumugam, T. (2020). MCO-linked domestic violence rises. New Straits Times. https://www.nst.com.my/news/exclusive/2020/04/581233/mco-linkeddomestic-violence-rises

CDC. (2019). How COVID-19 Spreads. Centers for Disease Control and Prevention. http://cdc.gov/2019-ncov/symptomstesting/symptoms.html

Dalton, C. B., Corbett, S. J., \& Katelaris, A. L. (2020). Pre-emptive low-cost social distancing and enhanced hygiene implemented before local COVID-19 transmission could decrease the number and severity of cases. The Medical Journal of Australia 212(10), 1-10. https://www.mja.com.au/journal/2020/pre-emptive-low-cost-social-distancing-and-enhancedhygiene-implemented-local-covid-19

Du, K. L., \& Swamy, M. N. S. (2013). Neural Network and Statistical Learning. Concordia University/ Montreal.

Fausset, L. (1994). Fundamental of Neural Networks: Architectures, Algorithms, and Applications. Prentice-Hall/ New York. FMT. (2020). Social distancing: How to do it right. Free Malaysia Today. https://www.freemalaysiatoday.com/c ategory/leisure/2020/03/26/socialdistancing-how-to-do-it-right/

Foo, L. P., Chin, M. Y., Tan, K. L., \& Phuah, K. T. (2020). The impact of COVID-19 on tourism industry in Malaysia. Current Issues in Tourism, 1-5. https://doi.org/10.1080/13683500.2020.1777951

Haque, A., Karim, W., Kabir, S. M. H., \& Tarofder, A. K. (2020). Understanding Social Distancing Intention among University Students during Covid-19 Outbreak: An Application of Protection Motivation Theory. TEST Engineering and Management 83(5), 16360-16377. http://irep.iium.edu.my/90965/ 
Huang, G. B., Zhu, Q. Y., \& Siew, C. K. (2004). Extreme learning machine: a new learning scheme of feedforward neural networks. IEEE International Joint Conference on Neural Networks 2, 25-29. https://doi.org/ 10.1109/IJCNN.2004.1380068

Huang, N. E., Qiao, F., \& Tung, K. (2020). A data-driven model for predicting the course of COVID-19 epidemic with applications for China, Korea, Italy, Germany, Spain, UK, and USA. medRxiv (preprint). https://doi.org/10.1101/2020.03.28.20046177

Hyndman, R. J., \& Athanasopoulos, G. (2018). Forecasting: Principles and Practice. OTexts/ Melbourne.

Khoo, L. S., Hasmi, A. H., Ibrahim, M. A., \& Mahmood, M. S. (2020). Management of the dead during COVID-19 outbreak in Malaysia. Forensic Science, Medicine, and Pathology (June 9), 1-8. https://doi.org/10.1007/s12024-020-00269-6.

Liang, N., \& Huang, G. (2006). A fast and accurate online sequential learning algorithm for a feed-forward network. IEEE Trans Neural Network 17(6), 1411-1423. https://doi.org/10.1109/TNN.2006.880583

Mahase E. (2020). Covid-19: UK starts social distancing after new model points to 260000 potential deaths. BMJ (Clinical research ed.), 368, m1089. https://doi.org/10.1136/bmj.m1089

McCulloch, W. S., \& Pits, W. H. (1943). A logical calculus of the ideas immanent in nervous activity. Bulletin of Mathematical Biophysics 5, 115-133. https://doi.org/10.1007/BF02478259

Moriyama, M., Walter, J. H., \& Akiko, I. (2020). Seasonality of Respiratory Viral Infections. Annual Reviews of Virology 7 , 83-101. https://doi.org/10.1146/annurev-virology-012420-022445

Plummer, E. A. (2000). Time Series Forecasting with Feed-Forward Neural Networks: Guidelines And Limitations. [Thesis]. Department of Computer Science and The Graduate School of The University of Wyoming.

PMO. (2020). 2020 economic stimulus package - PRIHATIN. Prime Minister's Office. https://www.pmo.gov.my/2020/03/pakejrangsangan-ekonomi-prihatin-rakyat-prihatin/.

Pontoh R. S., Toharudin, T., Zahroh, S., \& Supartini, E. (2020a). Effectiveness of the Public Health Measures to Mention the Spread of COVID-19. Communication and Mathematical Biology and Neuroscience, 31. https://doi.org/10.28919/cmbn/4711

Pontoh, R. S., Zahroh, S., Hidayat, Y., Aldella, R., \& Jiwani, N. M. (2020b). Covid-19 modelling in South Korea using a time series approach. International Journal of Advanced Science Technology 29(7), 1620-1632. https://sersc.org/journals/index.php/IJAST/article/view/16246

Pontoh, R. S., Zahroh, S., Akbar, A. A., Jiwani, N. M., \& Sunengsih, N. (2021a). Children Mental Health in Bandung During Covid-19 Pandemic: A Cross-Sectional Study. Communication and Mathematical Biology and Neuroscience, 31. https://doi.org/10.28919/cmbn/5631

Pontoh, R. S., Zahroh, S., \& Sunengsih, N. (2021b). New Normal Policy on the Rupiah Exchange Rate Using Long Short Term Memory. Journal of Physics: Conference Series 1863(2021), 012063. https://doi.org/ 10.1088/1742$6596 / 1863 / 1 / 012063$

Rettner, R. (2020). Up to 25\% of People with COVID-19 may not Show Symptoms. Livescience. https://www.livescience.com/coronavirus-asymptomatic-spread.html

Rosenblatt, F. (1962). Principles of Neurodynamics: Perceptrons and the Theory of Brain Mechanisms. Spartan/ Washington D.C.

Toharudin, T., Pontoh, R. S., Caraka, R.E., Zahroh, S., Kendogo, P., Sijabat, N., Sari, M.D.P., Gio, P.U., Basyuni, M., \& Pardamean, B. (2021). National Vaccination and Local Intervention Impacts on COVID-19 Cases. Sustainability $13,8282$. https://doi.org/10.3390/su13158282

Toharudin, T., Pontoh, R. S., Caraka, R. E., Zahroh, S., Youngjo L., \& Chen, R. C. (2021). Employing long short-term memory and Facebook prophet model in air temperature forecasting. Communications in Statistics - Simulation and Computation, 1-12. https://doi.org/10.1080/03610918.2020.1854302

Wang, S. J., Chen, H. L., Yan, W. J., Chen, Y. H., \& Fu, X. (2014). Face recognition and micro-expression recognition based on discriminant tensor subspace analysis plus extreme learning machine. Neural Processing Letters 39(1), $25-43$. https://doi.org/10.1007/s11063-013-9288-7

WHO. (2020). Situation Report-77: Coronavirus disease 2019 (COVID-19). World Health Organization. https://www.who.int/emergencies/diseases/novel-coronavirus-2019/situation-reports.

Worldometer. (2020). COVID-19 Coronavirus Pandemic. Worldometer. http://worldometers.info/coronavirus.

Xiao, D., Li, B., \& Mao, Y. (2017). A multiple hidden layers extreme learning machine method and its application. Hindawi Mathematical Problems in Engineering 2017, 1-11. https://doi.org/10.1155/2017/4670187 


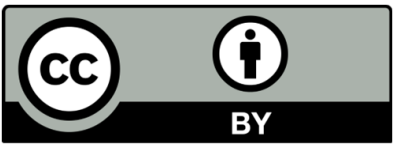

(C) 2022 by the authors; licensee Growing Science, Canada. This is an open access article distributed under the terms and conditions of the Creative Commons Attribution (CCBY) license (http://creativecommons.org/licenses/by/4.0/). 